\title{
Revisiting large neutrino magnetic moments
}

\author{
Manfred Lindner, Branimir Radovčić and Johannes Welter \\ Max-Planck-Institut für Kernphysik, \\ Saupfercheckweg 1, 69117 Heidelberg, Germany \\ E-mail: lindner@mpi-hd.mpg.de, radovcic@mpi-hd.mpg.de, \\ welter@mpi-hd.mpg.de
}

ABSTRACT: Current experimental sensitivity on neutrino magnetic moments is many orders of magnitude above the Standard Model prediction. A potential measurement of nextgeneration experiments would therefore strongly request new physics beyond the Standard Model. However, large neutrino magnetic moments generically tend to induce large corrections to the neutrino masses and lead to fine-tuning. We show that in a model where neutrino masses are proportional to neutrino magnetic moments. We revisit, discuss and propose mechanisms that still provide theoretical consistent explanations for a potential measurement of large neutrino magnetic moments. We find only two viable mechanisms to realize large transition magnetic moments for Majorana neutrinos only.

Keywords: Beyond Standard Model, Neutrino Physics

ARXIV EPRINT: 1706.02555 


\section{Contents}

1 Introduction 1

2 Naturalness bounds $\quad 2$

2.1 New physics above the electroweak scale 2

2.2 New physics below the electroweak scale 3

3 Natural large NMM via millicharged particles 4

4 Radiative neutrino mass model 5

5 Naturally large NMM via symmetries $\quad 7$

5.1 BFZ model 8

$\begin{array}{lll}5.2 & \text { Voloshin-type symmetry } & 10\end{array}$

$\begin{array}{ll}5.3 \text { Horizontal symmetry } & 10\end{array}$

6 Discussion and conclusion $\quad 11$

\section{Introduction}

The neutrino magnetic moment $(\mathrm{NMM})$ in the Standard Model $(\mathrm{SM})^{1}$ is of the order $10^{-19} \mu_{B}[1-5]$, where $\mu_{B}=\frac{e}{2 m_{e}}$ is the Bohr magneton. At the same time reactor, accelerator and solar neutrino experiments as well as astrophysical observations are lacking many orders of magnitude in sensitivity in order to test the small SM prediction (for a recent review see [6]). The best current laboratory limit is given by GEMMA, an experiment measuring the electron recoil of antineutrino-electron scattering near the reactor core. It constrains the effective magnetic moment to be less than $2.9 \cdot 10^{-11} \mu_{B}$ [7]. A recent study by Cañas et al. [8] showed that results of the solar neutrino experiment Borexino give similar limits. They obtain for the individual Majorana transition moments in the mass basis $\left|\Lambda_{1}\right| \leq 5.6 \cdot 10^{-11} \mu_{B},\left|\Lambda_{2}\right| \leq 4.0 \cdot 10^{-11} \mu_{B},\left|\Lambda_{3}\right| \leq 3.1 \cdot 10^{-11} \mu_{B}$.

On the other hand, the smallness of the SM prediction imply that a non-zero measurement of NMM would be a clear indication for new physics beyond the SM. In view of upcoming experiments, that are able to further increase the sensitivity on the NMM, it is worthy to ask what kind of new physics could explain large NMM. In other words, we want to address the question of how to generate large NMM in a theoretically consistent way.

The paper is organized as follows. In section 2 we review model independent bounds on the NMM from corrections to the neutrino mass. In section 3 we consider a model with

\footnotetext{
${ }^{1}$ In the pure SM neutrinos are massless and therefore the NMM is zero. Here we refer to the extensions of the SM allowing for neutrino masses.
} 
light millicharged particles. In section 4 we explicate the generic difficulty to obtain a large NMM without fine-tuning neutrino masses in a particularly insightful model. In section 5 we revisit and update constraints on existing models that successfully avoid fine-tuning. We discuss and conclude in section 6 .

\section{Naturalness bounds}

\subsection{New physics above the electroweak scale}

Since neutrinos are neutral, the leading contribution to the NMM is given by quantum corrections. Consider a theory with new physics at the scale $\Lambda$ and new couplings $G$ that introduces the NMM at 1-loop. The Feynman diagram generating the NMM $\mu_{\nu}$ for Majorana neutrinos is depicted in figure 1(a). Removing the photon line will directly result in a radiative neutrino mass correction $\delta m_{\nu}$ from the diagram in figure $1(\mathrm{~b})$. With the new physics above the electroweak scale, the effective NMM operator in the case of Majorana neutrino is of dimension seven and the effective mass operator is of dimension five. The generic estimate thus gives

$$
\mu_{\nu} \sim \frac{Q e G v_{H}^{2}}{\Lambda^{3}}, \quad \delta m_{\nu} \sim G \frac{v_{H}^{2}}{\Lambda}
$$

leading to

$$
\frac{\delta m_{\nu}}{0.1 \mathrm{eV}} \sim \frac{1}{Q}\left(\frac{\mu_{\nu}}{10^{-19} \mu_{B}}\right)\left(\frac{\Lambda}{\mathrm{TeV}}\right)^{2}
$$

where $v_{H}$ is the vacuum expectation value of the Higgs and $Q$ is the charge of the particles running inside the loop in units of the electron charge $e$. To avoid fine-tuning, the radiative neutrino mass correction should not be larger than the measured neutrino masses, $\delta m_{\nu} \lesssim$ $m_{\nu}$. Using reasonable numbers, $m_{\nu} \sim 0.1 \mathrm{eV}, \Lambda \sim \mathrm{TeV}$ and $Q \sim 1$ we obtain the naive limit

$$
\mu_{\nu} \lesssim 10^{-19} \mu_{B}
$$

For Dirac neutrinos the 1-loop effective NMM and neutrino mass operators are of dimension six and four respectively. With diagrams similar to figure 1 this leads to

$$
\mu_{\nu} \sim \frac{Q e G v_{H}}{\Lambda^{2}}, \quad \delta m_{\nu} \sim G v_{H}
$$

By taking the ratio $\delta m_{\nu} / \mu_{\nu}$ we get the same constraint as in eqs. (2.2) and (2.3).

The current best laboratory experimental limit for the NMM is at $\mu_{\nu} \sim 2.9 \cdot 10^{-11} \mu_{B}$ [7], while neutrino masses above $0.2 \mathrm{eV}$ are in conflict with cosmological observations [9]. Therefore the above estimate shows that generating large NMM while simultaneously keeping the radiative mass correction $\delta m_{\nu}$ low, requires a significant amount of fine-tuning. To reach values $\mu_{\nu} \gtrsim 10^{-12} \mu_{B}$, which will be probed in future experiments [10-13], fine-tuning of seven orders of magnitude is required.

If the contribution to neutrino masses from the diagram in figure 1(b) is suppressed for some reason, there are still contributions from higher-loop diagrams induced by the 


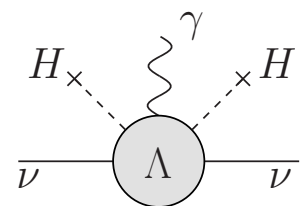

(a)

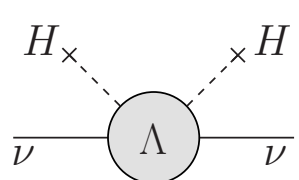

(b)

Figure 1. Feynman diagrams generating the NMM and the radiative neutrino mass for Majorana neutrinos induced by new physics above the electroweak scale.

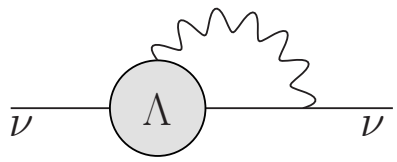

Figure 2. Higher-loop neutrino mass contribution induced by the presence of the NMM operator.

NMM operator like the one in figure 2. In order to derive constraints on the NMM, Bell et al. [14, 15] and Davidson et al. [16] performed effective operator analyses for Dirac and Majorana neutrinos. Requiring the naturalness condition $\delta m_{\nu} \lesssim m_{\nu}$ to avoid the fine-tuning they found the model independent bound for Dirac neutrinos of the order $\mu_{\nu} \lesssim 10^{-15} \mu_{B}$, when taking the new physics scale $\Lambda=1 \mathrm{TeV}$ and $\delta m_{\nu} \lesssim 0.2 \mathrm{eV}$ [14].

A similar analysis for Majorana neutrinos $[15,16]$ shows more room for large NMMs. The reason is that for Majorana neutrinos the NMM operator is flavour antisymmetric while the mass operator is flavour symmetric. For $\Lambda=1 \mathrm{TeV}$ and $m_{\nu} \lesssim 0.3 \mathrm{eV}$, they obtain the model independent limits $\mu_{\nu_{\tau} \nu_{\mu}}, \mu_{\nu_{\tau} \nu_{e}} \lesssim 10^{-9} \mu_{B}, \mu_{\nu_{\mu} \nu_{e}} \lesssim 3 \cdot 10^{-7} \mu_{B}$ [15], which are already worse than current experimental constraints.

\subsection{New physics below the electroweak scale}

Now let us assume that the new physics is generated below the electroweak scale. For example one could think of a hidden sector, containing light particles. In this case, the effective NMM and neutrino mass operators generated by the Feynman diagrams in figure 3 are of dimension five and three respectively. The naive estimate

$$
\mu_{\nu} \sim \frac{Q e G}{\Lambda}, \quad \delta m_{\nu} \sim G \Lambda
$$

leads to

$$
\frac{\delta m_{\nu}}{0.1 \mathrm{eV}} \sim \frac{1}{Q}\left(\frac{\mu_{\nu}}{10^{-13} \mu_{B}}\right)\left(\frac{\Lambda}{\mathrm{GeV}}\right)^{2} .
$$

Given the estimates of eqs. (2.2) and (2.6) it seems that there are two possibilities for generating large NMM. Either the masses of the new particles are high and one has to find a mechanism that avoids the naturalness bound or the new particles are light with fractional charge $Q<1$. In the next section we want to address the latter case, while for the rest of the paper we will assume that new physics is above the electroweak scale. 


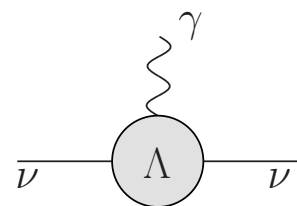

(a)

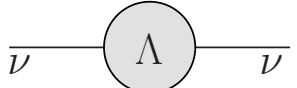

(b)

Figure 3. The Feynman diagrams for the NMM and the radiative neutrino mass induced by new physics below the electroweak scale.

\section{$3 \quad$ Natural large NMM via millicharged particles}

Motivated by the estimate of eq. (2.6) we are interested in particles with low mass, $\Lambda<1 \mathrm{GeV}$, and fractional charge $Q$ as large as possible, while satisfying the current phenomenological bounds on millicharged particles. For example, if we would have $Q \sim 0.1$ and $\Lambda \sim 0.1 \mathrm{GeV}$ the estimate shows that one could reach $\mu_{\nu} \sim 10^{-12} \mu_{B}$ in a technically natural way.

In order to investigate this on a more quantitative level, we assume a millicharged scalar $s$ and a Dirac fermion $\psi$ coupling to light Majorana neutrinos in the form

$$
\mathcal{L}=f_{i} \overline{\psi_{R}} \nu_{L i} s+f_{j}^{\prime} \overline{\nu_{L j}} \psi_{L} s^{\dagger}+\text { h.c. }
$$

Such couplings generate both, corrections to the neutrino masses as well as NMMs. In this work we compute the loop diagrams with the help of package $\mathrm{X}$ [17]. For the neutrino mass correction we obtain in the limit $M \equiv m_{s}=m_{\psi}$

$$
\delta m_{\nu_{i} \nu_{j}}=\frac{f_{i} f_{j}^{\prime}+f_{j} f_{i}^{\prime}}{16 \pi^{2}} M \log \frac{M^{2}}{\mu^{2}} .
$$

The magnetic and electric dipole moments can be extracted from the corresponding form factors of the effective neutrino-photon interaction Lagrangian

$$
\mathcal{L}_{\text {int }}^{\mathrm{eff}}=-\frac{1}{2} \mathcal{F}_{\mu}^{j i}\left(q^{2}\right) \overline{\nu_{j}} \frac{i \sigma_{\mu \nu} q^{\nu}}{m_{\nu_{j}}+m_{\nu_{i}}} \nu_{i}-\frac{i}{2} \mathcal{F}_{\epsilon}^{j i}\left(q^{2}\right) \overline{\nu_{j}} \frac{i \sigma_{\mu \nu} q^{\nu}}{m_{\nu_{j}}+m_{\nu_{i}}} \gamma_{5} \nu_{i}
$$

by taking the limit $q^{2} \rightarrow 0$

$$
\begin{aligned}
\mu_{\nu_{j} \nu_{i}} & =\mathcal{F}_{\mu}^{j i}(0), \\
\epsilon_{\nu_{j} \nu_{i}} & =\mathcal{F}_{\epsilon}^{j i}(0) .
\end{aligned}
$$

Projecting out the corresponding form factors, we get in the limit $M \equiv m_{s}=m_{\psi}$

$$
\begin{aligned}
\mu_{\nu_{j} \nu_{i}} & =\frac{i Q e}{32 \pi^{2} M} \operatorname{Im}\left[f_{i} f_{j}^{\prime}-f_{j} f_{i}^{\prime}\right], \\
\epsilon_{\nu_{j} \nu_{i}} & =\frac{i Q e}{32 \pi^{2} M} \operatorname{Re}\left[f_{i} f_{j}^{\prime}-f_{j} f_{i}^{\prime}\right],
\end{aligned}
$$




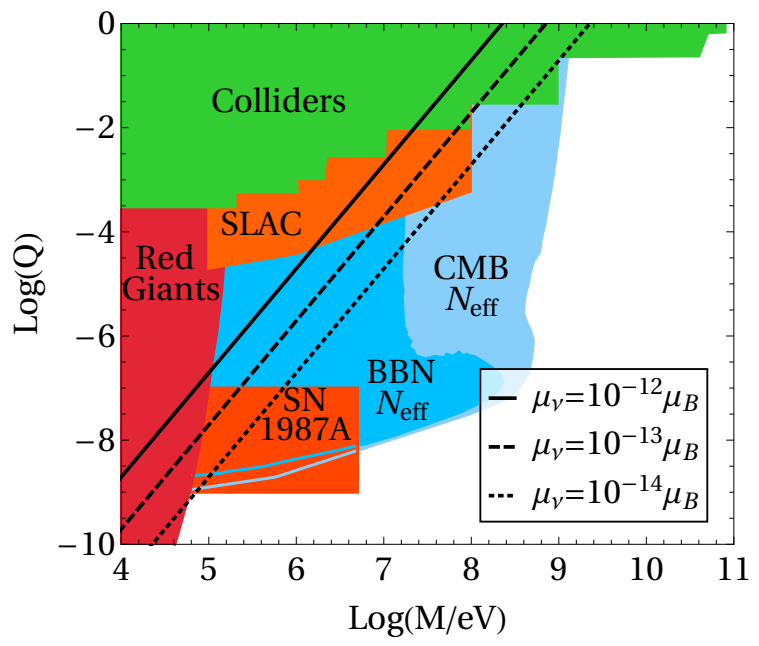

Figure 4. Lines of constant $\mu_{\nu}$ for $\delta m_{\nu}=0.2 \mathrm{eV}$ in the plane of mass $M$ and fractional charge $Q$ of the millicharged particle. The constraints are coming from several observables and are taken from ref. [18]. See also the working group report and references therein [19].

where $Q$ is the fractional charge of $s$ and $\psi$. Assuming no cancellation in the couplings among the flavours one arrives at the relation between $Q$ and $M$

$$
\frac{\mu_{\nu}}{\delta m_{\nu}}=\frac{Q e}{4 M^{2}}
$$

Now one can ask the question, which values for mass and millicharge of the new particles are necessary so that observable NMMs can be generated without fine-tuning. Taking $\delta m_{\nu} \sim 0.2 \mathrm{eV}$ and assuming values of $\mu_{\nu}$ close to the current experimental sensitivity, we obtain the required ratio $Q / M^{2}$. The result is shown in figure 4 , where we overlay the curves of constant NMM over excluded regions [18, 19] in the plane of fractional charge and mass of the new particle. For milicharged particles in dark matter context, see also [20].

There seems to be no room for large NMMs generated by light millicharged particles.

\section{Radiative neutrino mass model}

Let us now explicate the generic difficulty to obtain large NMMs without fine-tuning neutrino masses in models with new physics above the electroweak scale. We start by adding two scalar $\mathrm{SU}(2)_{L}$ doublets $\eta, \phi$ as well as a new charged Dirac fermion $\Sigma=\Sigma_{L}+\Sigma_{R}$ with the quantum numbers

$$
\begin{aligned}
\eta & =\left(\begin{array}{c}
\eta^{0} \\
\eta^{-}
\end{array}\right) \sim(2,-1 / 2), & L_{L i}=\left(\begin{array}{c}
\nu_{L i} \\
l_{L i}
\end{array}\right) \sim(2,-1 / 2), \\
\phi & =\left(\begin{array}{c}
\phi^{-} \\
\phi^{--}
\end{array}\right) \sim(2,-3 / 2), & \Sigma_{L / R}^{-} \sim(1,-1),
\end{aligned}
$$




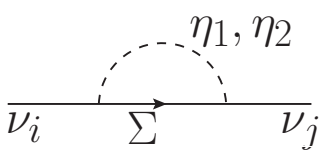

(a)

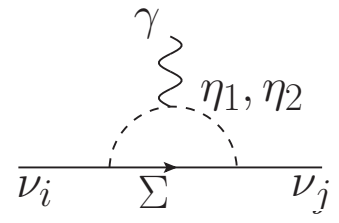

(b)

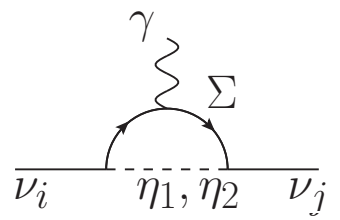

(c)

Figure 5. Diagrams for neutrino mass and magnetic moment in the radiative neutrino mass model.

where $L_{L i}$ is the SM lepton doublet. Neutrinos are massless at the tree-level and neutrino masses are generated at loop-level via the Yukawa interactions

$$
\mathcal{L}_{Y}=Y_{i} \overline{\Sigma_{R}} \tilde{\eta}^{\dagger} L_{L i}+Y_{j}^{\prime} \overline{\Sigma_{L}^{c}} \phi^{\dagger} L_{L j}+\text { h.c. }
$$

From the scalar potential interactions the electroweak symmetry breaking generates the mixing between $\eta^{-}$and $\phi^{-}$

$$
\left(\begin{array}{l}
\eta_{1} \\
\eta_{2}
\end{array}\right)=\left(\begin{array}{cc}
\cos \theta & \sin \theta \\
-\sin \theta & \cos \theta
\end{array}\right)\left(\begin{array}{l}
\eta \\
\phi
\end{array}\right)
$$

which leads to

$$
\mathcal{L}_{Y}=Y_{i} \overline{\Sigma_{R}}\left(\cos \theta \eta_{1}^{-}-\sin \theta \eta_{2}^{-}\right) \nu_{L i}+Y_{j}^{\prime} \overline{\nu_{L j}^{C}}\left(\sin \theta \eta_{1}^{+}+\cos \theta \eta_{2}^{-}\right) \Sigma_{L}+\text { h.c. }
$$

The neutrino mass matrix results from the loop diagram depicted in figure 5 (a). Note that the contributions from $\eta_{1}$ and $\eta_{2}$ differ by a relative minus sign, so that the divergencies cancel each other. We obtain

$$
M_{\nu_{i} \nu_{j}}=\frac{Y_{i} Y_{j}^{\prime}+Y_{j} Y_{i}^{\prime}}{16 \pi^{2}} m_{\Sigma} \sin \theta \cos \theta\left[\frac{m_{\eta_{1}}^{2}}{m_{\eta_{1}}^{2}-m_{\Sigma}^{2}} \log \left(\frac{m_{\eta_{1}}^{2}}{m_{\Sigma}^{2}}\right)-\frac{m_{\eta_{2}}^{2}}{m_{\eta_{2}}^{2}-m_{\Sigma}^{2}} \log \left(\frac{m_{\eta_{2}}^{2}}{m_{\Sigma}^{2}}\right)\right] .
$$

We added only one charged Dirac fermion $\Sigma$, implying that only two of the eigenvalues of $M$ are non-zero. Hence the lightest neutrino is massless.

The electric and magnetic dipole moments result from the diagrams depicted in figure 5(b), (c) and are computed as in the previous section. The result is

$$
\begin{aligned}
\mu_{\nu_{j} \nu_{i}} & =\frac{-i e \sin \theta \cos \theta}{16 \pi^{2} m_{\Sigma}} \operatorname{Im}\left[Y_{i} Y_{j}^{\prime}-Y_{j} Y_{i}^{\prime}\right] f\left(\frac{m_{1}^{2}}{m_{\Sigma}^{2}}, \frac{m_{2}^{2}}{m_{\Sigma}^{2}}\right), \\
\epsilon_{\nu_{j} \nu_{i}} & =\frac{-i e \sin \theta \cos \theta}{16 \pi^{2} m_{\Sigma}} \operatorname{Re}\left[Y_{i} Y_{j}^{\prime}-Y_{j} Y_{i}^{\prime}\right] f\left(\frac{m_{1}^{2}}{m_{\Sigma}^{2}}, \frac{m_{2}^{2}}{m_{\Sigma}^{2}}\right),
\end{aligned}
$$

with the loop function

$$
f\left(a_{1}, a_{2}\right)=\frac{a_{1}\left(a_{2}-1\right)^{2} \log \left(a_{1}\right)-\left(a_{1}-1\right)\left(-\left(a_{1}+1\right) a_{2}+\left(a_{1}-1\right) a_{2} \log \left(a_{2}\right)+a_{1}+a_{2}^{2}\right)}{\left(a_{1}-1\right)^{2}\left(a_{2}-1\right)^{2}} .
$$


Note that for Majorana neutrinos, we expect $\mu_{\nu_{j} \nu_{i}}$ and $\epsilon_{\nu_{j} \nu_{i}}$ to be hermitian and antisymmetric, i.e. to be purely imaginary. In addition, if $\mathrm{CP}$ is conserved, either the magnetic or the electric moment is zero. See for example ref. [6] for more details. Now, what can we learn from this exercise?

To answer this question, let us first recognize that in this model the origin of the NMM is the same as the neutrino mass. There are no other sources of neutrino masses so that fine-tuning is not possible. Due to this connection it is possible to predict the NMM matrix by using experimental values of the leptonic mixing matrix and the neutrino masses.

As an example, we assume all CP-phases of the PMNS-matrix $U$ to be zero. Since in our model the lightest neutrino is massless, the masses of the other two are given by the measured mass square differences. We use the results of the global fit from ref. [21] and obtain the mass matrix from the relation

$$
M_{\nu_{j} \nu_{i}}=U \operatorname{diag}\left(0, m_{\nu_{2}}, m_{\nu_{3}}\right) U^{\dagger} .
$$

Using eq. (4.6) with reasonable numbers for the scalar and fermion masses $m_{1}=1.1 \mathrm{TeV}$, $m_{2}=0.9 \mathrm{TeV}, m_{\Sigma}=1 \mathrm{TeV}$ one can solve eq. (4.10) for the Yukawa couplings

$$
\left(\begin{array}{l}
Y_{1} \\
Y_{2} \\
Y_{3}
\end{array}\right)=\left(\begin{array}{c}
1 \\
2.1 \mp 1.6 i \\
0.7 \mp 2.8 i
\end{array}\right) \cdot x \cdot 10^{-6}, \quad\left(\begin{array}{c}
Y_{1}^{\prime} \\
Y_{2}^{\prime} \\
Y_{3}^{\prime}
\end{array}\right)=\left(\begin{array}{c}
2.9 \\
6.0 \pm 4.5 i \\
2.0 \pm 8.2 i
\end{array}\right) \cdot \frac{1}{x} \cdot 10^{-6}, \quad x \in \mathcal{C} .
$$

In this way we obtain for the Majorana neutrino electric and dipole moment matrices

$$
\mu_{\nu_{j} \nu_{i}}= \pm i\left(\begin{array}{ccc}
0 & -2 & -3.5 \\
2 & 0 & -5.9 \\
3.5 & 5.9 & 0
\end{array}\right) \cdot 10^{-21} \mu_{B}, \quad \quad \epsilon_{\nu_{j} \nu_{i}}=0
$$

with values many orders of magnitude below current experimental sensitivity. Since it does not allow for fine-tuning, this model illustrates the generic problem in generating large NMMs. Therefore, consistent models predicting large NMMs have to include a mechanism that avoids this connection of neutrino mass and NMM. That is why in well-studied models without such a mechanism, like the left-right symmetric model [22] and the supersymmetric model [23], the NMM predictions are far from being detected in next-generation experiments. On the other hand, a recent parameter study in the framework of the minimal supersymmetric model found room for large NMM [24], but does not solve the fine-tuning problem.

\section{$5 \quad$ Naturally large NMM via symmetries}

To generate a sizable NMM and to avoid fine-tuning by suppressing neutrino mass loop contributions one should rely on some sort of a symmetry. There are two classes of symmetries. First one could try to build a suppression mechanism using one of the quantum numbers of the photon. This was proposed by Barr, Freire and Zee (BFZ) in refs. [25-27] using the spin. For the other quantum numbers, like the parity or charge conjugation we 


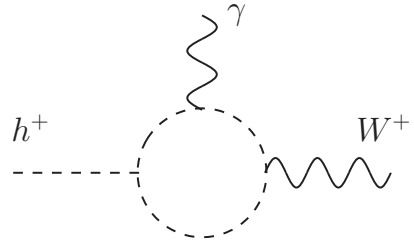

(a)

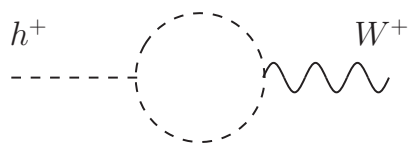

(b)

Figure 6. The sub-diagrams of the BFZ spin suppression mechanism. When removing the photon line, only the longitudinal components of the $W$ will contribute, because of the spin conservation.

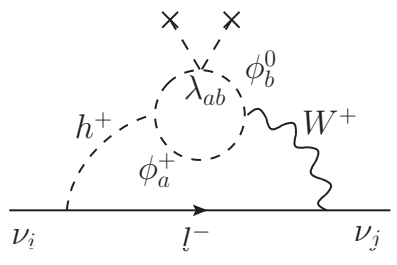

(a)

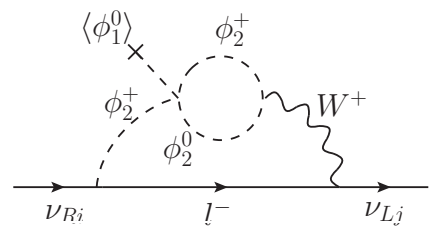

(b)

Figure 7. (a) Two-loop neutrino mass contribution in the BFZ model. The NMM can be computed by attaching the photon line to any of the charged particles inside the loop. (b) A similar diagram for the model with Dirac neutrinos.

checked all one loop subdiagram possibilities and found no such suppression mechanism. Second, there are models exploiting the symmetry properties of the effective NMM and mass operators. The following were already proposed in the literature, namely: Voloshintype symmetry [28, 29] (e.g. $\mathrm{SU}(2)$ with $\left.\nu \leftrightarrow \nu^{C}\right), \mathrm{SU}(2)$ horizontal symmetry [30, 31] and discrete symmetries [32-36].

\subsection{BFZ model}

In ref. [25] BFZ proposed the spin-suppression mechanism. The idea is that the loop diagram generating the NMM has a sub-diagram involving the scalar $h^{+}$and the vector $W$. The neutrino mass contribution diagram has the same sub-diagram with the photon line removed, see figure 6 . In this case, because of the spin conservation, only the longitudinal degrees of freedom of the $W$ contribute. When the sub-diagram is embeded in the full diagram in figure 7 (a) it will be proportional to the Yukawa coupling and the neutrino mass contribution is thus suppressed by powers of the lepton mass. Note that this mechanism still holds for higher order contributions, i.e. also diagrams of the form of figure 2 are suppressed. In this way the naturalness bounds summarized in the previous section can be avoided.

An essential ingredient for this mechanism is the charged scalar singlet $h^{+}$with the coupling to the SM lepton doublet in the form

$$
\mathcal{L}=f^{j i} h^{+} \overline{L_{L j}^{c}} i \tau_{2} L_{L i}
$$


The realization of spin suppression mechanism in [25] uses three scalar doublets $\phi_{a}$, with the neutral component of one of them, say $\phi_{1}$, obtaining a non-zero vacuum expectation value. From the antisymmetric interaction

$$
\mathcal{L}=\tilde{M}^{a b} h^{+}\left(\phi_{a}^{-} \phi_{b}^{0}-\phi_{b}^{-} \phi_{a}^{0}\right)
$$

and the quartic term of the scalar potential

$$
\mathcal{L}=\lambda_{a b}\left\langle\phi_{1}^{\dagger}\right\rangle \phi_{a}\left\langle\phi_{1}^{\dagger}\right\rangle \phi_{b}
$$

one obtains the diagram for the NMM, see figure 7(a).

In order to estimate if the model is still viable, one can derive the following relation between the radiative neutrino mass $\delta m_{\nu_{i} \nu_{j}}$ and the NMM $\mu_{\nu_{i} \nu_{j}}[25]$ :

$$
\delta m_{\nu_{i} \nu_{j}}=\left(\frac{m_{j}^{2}-m_{i}^{2}}{M_{W}^{2}}\right) \cdot\left(\frac{\delta M_{2}^{2}+\delta M_{3}^{2}}{2 M^{2}}\right) \cdot\left(\frac{M}{\mathrm{TeV}}\right)^{2} \cdot\left(\frac{\mu_{\nu_{i} \nu_{j}}}{10^{-12} \mu_{B}}\right) \cdot 0.5 \cdot 10^{6} \mathrm{eV},
$$

where $m_{i}$ are the charged lepton masses, $M_{W}$ the $\mathrm{W}$ boson mass, $M$ is the scalar mass, assuming $M \equiv M_{2} \sim M_{3}$ and $\delta M_{2}, \delta M_{3}$ being the mass differences of the charged and neutral components of $\phi_{2}$ and $\phi_{3}$. New charged scalar particles like $h^{+}$and $\phi_{2,3}^{+}$would have been seen by the LHC if considerably lighter than $1 \mathrm{TeV}$. See for example SUSY searches for slepton decays $[37,38]$. In the limit of massless neutralinos the bounds are of the same order of magnitude as for $h^{+}$due to similar decay channels. Let us therefore assume the new particle masses at $\mathrm{TeV}$ scale, $M \sim 1 \mathrm{TeV}$. For $\mu_{\nu_{i} \nu_{j}} \sim 10^{-12} \mu_{B}$ this yields

$$
\begin{aligned}
\delta m_{\nu_{e} \nu_{\mu}} & =\left(\frac{\delta M_{2}^{2}+\delta M_{3}^{2}}{2 M^{2}}\right) \mathrm{eV} \\
\delta m_{\nu_{\mu} \nu_{\tau}}, \delta m_{\nu_{\tau} \nu_{e}} & =\left(\frac{\delta M_{2}^{2}+\delta M_{3}^{2}}{2 M^{2}}\right) \cdot 2.5 \cdot 10^{2} \mathrm{eV} .
\end{aligned}
$$

In order to satisfy the limit on the upper bound of neutrino masses from various cosmological observations [9], one needs $\delta m_{\nu_{i} \nu_{j}} \lesssim m_{\nu_{i} \nu_{j}}<0.2 \mathrm{eV}$ and therefore $\frac{\delta M_{2}^{2}+\delta M_{3}^{2}}{2 M^{2}}<0.8 \cdot 10^{-3}$ with no need for fine-tuning. This shows that even though this is a two-loop diagram, the mechanism still gives sizable NMMs and is in agreement with current experimental bounds.

It is interesting to think about a modified version of this model in order to apply the idea to Dirac neutrinos. We hence need a scalar connecting the right-handed neutrinos and the left-handed charged leptons. Beside the Higgs doublet $\phi_{1}$, one could introduce an additional scalar doublet $\phi_{2}=\left(\phi_{2}^{0}, \phi_{2}^{-}\right)^{T}$ with the interaction $Y \overline{L_{L}} \phi_{2} \nu_{R}$. Then with the term from the scalar potential $\lambda \phi_{1}^{\dagger} \phi_{2} \phi_{2}^{\dagger} \phi_{2}$ one would obtain the Feynman diagram depicted in figure $7(\mathrm{~b})$ leading to a large NMM. However, the potential also contains the coupling $\lambda^{\prime} \phi_{2}^{\dagger} \phi_{1} \phi_{1}^{\dagger} \phi_{1}$ which after electroweak symmetry breaking generates a term linear in $\phi_{2}^{0}$, i.e. inducing $\left\langle\phi_{2}^{0}\right\rangle \neq 0$. This leads to an additional tree-level source of neutrino mass and thus fine-tuning can not be avoided. Therefore, there is no simple implementation of the BFZ spin suppression mechanism for Dirac neutrinos. 


\subsection{Voloshin-type symmetry}

Another suppression mechanism is to impose $\mathrm{SU}(2)_{\nu}$ symmetry with $\left(\left(\nu_{R}\right)^{C}, \nu_{L}\right)^{T}$ transforming as a doublet. It contains the transformation $\nu_{L} \rightarrow\left(\nu_{R}\right)^{C}, \nu_{R} \rightarrow-\left(\nu_{L}\right)^{C}$, so that the mass and the NMM operators transform as [28]

$$
\begin{aligned}
\overline{\nu_{L}} \nu_{R} & \rightarrow-\overline{\nu_{L}} \nu_{R}, \\
\overline{\nu_{L}} \sigma_{\mu \nu} \nu_{R} F^{\mu \nu} & \rightarrow+\overline{\nu_{L}} \sigma_{\mu \nu} \nu_{R} F^{\mu \nu},
\end{aligned}
$$

i.e. the NMM term is invariant under this symmetry, while the mass term is not. Note that for incorporating this idea one needs Dirac neutrinos. In an UV-complete theory $\left(\nu_{R}\right)^{C}$ then needs to be in the same multiplet with $\nu_{L}$, which is already a part of the $\mathrm{SU}(2)_{L}$ doublet. The simplest possible implementation is to enlarge the electroweak gauge symmetry to $\mathrm{SU}(3)_{L} \times \mathrm{U}(1)_{X}$ from ref. [29]. The $\mathrm{SU}(2)_{\nu}$ symmetry can not be exact and the neutrino mass is therefore proportional to the breaking scale of the new symmetry.

The NMM and neutrino mass are generated by diagrams with two charged components $\eta_{1}$ and $\eta_{2}$ from the scalar $\mathrm{SU}(3)_{L}$ triplet. They are related by [29]

$$
\mu_{\nu}=\delta m_{\nu} \frac{2 e}{\Delta m_{\eta}^{2}} \log \frac{m_{\eta}^{2}}{m_{\tau}^{2}}
$$

We have to take into account the naturalness condition on the squared mass difference $\Delta m_{\eta}^{2}=m_{\eta_{1}}^{2}-m_{\eta_{2}}^{2}$, emerging from radiative corrections after symmetry breaking [29]:

$$
\Delta m_{\eta}^{2} \gtrsim \frac{\alpha_{W}}{4 \pi} M_{V}^{2}
$$

where $M_{V}$ is the mass of the vector boson associated with the $\mathrm{SU}(2)_{\nu}$ symmetry breaking and $\alpha_{W}$ is the electroweak fine-structure constant.

Taking the experimental limits on the $\mathrm{SU}(3)_{L}$ gauge boson masses [39] into consideration we set $M_{V} \sim m_{\eta} \sim 5 \mathrm{TeV}$ and get $\Delta m_{\eta}^{2} \gtrsim 7 \cdot 10^{5} \mathrm{GeV}^{2}$. By setting $\delta m_{\nu} \lesssim 0.2 \mathrm{eV}$ from eq. (5.9) we obtain $\mu_{\nu} \lesssim 10^{-16} \mu_{B}$. This still implies fine-tuning of four orders of magnitude to reach an observable NMM of $\mu_{\nu} \sim 10^{-12} \mu_{B}$. We thus conclude that within this framework it is not possible to generate observable NMM in theoretically consistent way.

\subsection{Horizontal symmetry}

The idea from the Voloshin symmetry can also be applied to Majorana neutrinos, which have zero diagonal NMM. Babu and Mohapatra [30] proposed that a large transition NMM can be achieved while suppressing neutrino mass contribution by using horizontal flavour $\mathrm{SU}(2)_{H}$ symmetry. In their model the electron and muon $\mathrm{SU}(2)_{L}$ doublets together form the $\mathrm{SU}(2)_{H}$ doublet $\Psi_{L}$, while the tau doublet is a $\mathrm{SU}(2)_{H}$ singlet $\Psi_{3 L}$. Also the righthanded electron and muon together form a $\mathrm{SU}(2)_{H}$ doublet $\Psi_{R}$.

For this mechanism to work, Babu and Mohapatra introduce in addition to the Higgs doublet $\phi_{s}$ the following new scalars: one bidoublet $\phi$ (i.e. doublet under $\mathrm{SU}(2)_{H}$ as well as under $\left.\mathrm{SU}(2)_{L}\right)$, one $\mathrm{SU}(2)_{H}$ doublet $\eta=\left(\eta_{1}^{+} \eta_{2}^{+}\right)$and two $\mathrm{SU}(2)_{H}$ triplets $\sigma_{1,2}$. The latter are responsible for breaking the horizontal symmetry in such a way that there is no 
tree-level mixing between generation-changing horizontal gauge bosons and the generationdiagonal ones, for more details we refer to ref. [30].

Introducing this set of particles lead among others to the Yukawa couplings $f \eta i \tau_{2} \overline{\Psi_{L}^{c}} i \tau_{2} \Psi_{3 L}$ and $f^{\prime} \operatorname{tr}\left(\overline{\Psi_{L}} \phi\right) \tau_{R}$. Together with the interaction $\mu_{1} \kappa_{s}\left(\eta_{1}^{+} \phi_{1}^{+}+\eta_{2}^{+} \phi_{2}^{+}\right)$coming from the cubic term from the scalar potential, where $\kappa_{s}$ is the vacuum expectation value of the SM Higgs, one arrives at the $\nu_{e}-\nu_{\mu}$ transition NMM

$$
\mu_{\nu_{e} \nu_{\mu}}=2 e \frac{f f^{\prime}}{16 \pi^{2}} m_{\tau} \frac{\mu_{1} \kappa_{s}}{m_{\eta}^{2}-m_{\phi}^{2}}\left(\frac{1}{m_{\eta}^{2}}-\frac{1}{m_{\phi}^{2}}\right),
$$

with $m_{\eta}=m_{\eta_{1}}=m_{\eta_{2}}$ and $m_{\phi}=m_{\phi_{1}}=m_{\phi_{2}}$. The horizontal symmetry is spontaneously broken by the vacuum expectation values of the scalar triplets. The breaking induces a mass splitting between the charged components of $\phi$ and $\eta$ and thus leads to non-zero neutrino mass

$$
\delta m_{\nu_{e} \nu_{\mu}}=\frac{f f^{\prime}}{16 \pi^{2}} m_{\tau} \mu_{1} \kappa_{s}\left(\frac{1}{m_{\phi_{1}}^{2}-m_{\eta_{1}}^{2}} \log \frac{m_{\phi_{1}}^{2}}{m_{\eta_{1}}^{2}}-\frac{1}{m_{\phi_{2}}^{2}-m_{\eta_{2}}^{2}} \log \frac{m_{\phi_{2}}^{2}}{m_{\eta_{2}}^{2}}\right) .
$$

Assuming $\Delta m_{\eta}^{2}=m_{\eta_{2}}^{2}-m_{\eta_{1}}^{2} \ll m_{\eta}^{2}$ and $\Delta m_{\phi}^{2}=m_{\phi_{2}}^{2}-m_{\phi_{1}}^{2} \ll m_{\phi}^{2}$ as well as $\Delta m_{\eta}^{2} / m_{\eta}^{2}=$ $\Delta m_{\phi}^{2} / m_{\phi}^{2}$ one obtains

$$
\left(\frac{\mu_{\nu_{e} \nu_{\mu}}}{10^{-12} \mu_{B}}\right)=2\left(\frac{\delta m_{\nu_{e} \nu_{\mu}}}{\mathrm{eV}}\right)\left(\frac{\mathrm{GeV}^{2}}{\Delta m_{\eta}^{2}}\right)\left(\frac{m_{\eta}^{2}}{m_{\phi}^{2}}-1\right) \log \frac{m_{\eta}^{2}}{m_{\phi}^{2}} .
$$

This shows that one can obtain NMM of the order $10^{-12} \mu_{B}$ without fine-tuning, if the mass splitting $\Delta m_{\eta}^{2}$ is at GeV scale. The $\Delta m_{\eta}^{2}$ can be small and technically natural because it emerges from a soft cubic interaction with the triplet $\sigma_{1}$ that breaks the $\mathrm{SU}(2)_{H}$.

The model can accommodate $\mathrm{SU}(2)_{H}$ breaking in the charged lepton $m_{e}$ and $m_{\mu}$ masses. It also predicts additional neutrino mass contributions $m_{\nu_{e} \nu_{\tau}}$ and $m_{\nu_{\mu} \nu_{\tau}}$. Demanding that their values are less than $0.2 \mathrm{eV}$ as well as requiring that the charged lepton masses are reproduced, leads to constraints on the coupling constants. However, we have checked that choosing new physics scale at $\mathrm{TeV}$ and couplings of order one still allows for $\mu_{\nu_{e} \nu_{\mu}} \sim 10^{-12} \mu_{B}$

One could think of including the $\tau$ flavour instead of $e$ or $\mu$ flavour in $\mathrm{SU}(2)_{H}$, or extending the horizontal symmetry to all three generations, e.g. using $\mathrm{SU}(3)_{H}$. Both of which would not allow for an extra source of the horizontal symmetry breaking in the coupling of the Higgs boson to charged leptons, since $h \rightarrow \tau \tau$ decays have been observed by the LHC [40, 41]. This mechanism therefore can only give a large $\nu_{e}-\nu_{\mu}$ transition moment.

\section{Discussion and conclusion}

SM predictions for the NMM are many orders of magnitude lower than current experimental sensitivity. With large NMMs generated by millicharged particles below the electroweak scale one can in principle avoid fine-tuning of the neutrino masses, but it would be in 
strong tension with cosmological observations. As we have showed in a very insightful model, theories with new physics above the electroweak scale predicting observable NMMs generically lead to large neutrino mass corrections, thus requiring fine-tuning of several orders of magnitude. We reviewed models proposed in literature that avoid the resulting naturalness bounds and suppress the neutrino mass correction by a symmetry. It turned out that building a model with large Dirac NMM in a technically natural way does not seem to be possible anymore. On the other hand, for Majorana neutrinos, using a $\mathrm{SU}(2)_{H}$ horizontal symmetry one can only realize a large $\nu_{e}-\nu_{\mu}$ transition moment. In the BFZ model, which relies on the spin-suppression mechanism, it is also possible to generate sizable $\nu_{e}-\nu_{\mu}$ as well as $\nu_{e}-\nu_{\tau}$ and $\nu_{\mu}-\nu_{\tau}$ transition moments.

In ref. [42] Frère, Heeck and Mollet derive inequalities between the transition moments for Majorana neutrinos. They argue that a possible measurement of $\mu_{\nu_{\tau}}$ at SHiP [43] would hint to the Dirac nature of the neutrino. If SHiP would indeed measure a sizable $\mu_{\nu_{\tau}}$, it would therefore require new ideas how to accommodate large Dirac NMMs while avoiding fine-tuned neutrino masses.

\section{Acknowledgments}

We are thankful for very helpful discussions with Evgeny Akhmedov, Hiren Patel and Stefan Vogl. BR acknowledges the support by the Alexander von Humboldt Foundation.

Open Access. This article is distributed under the terms of the Creative Commons Attribution License (CC-BY 4.0), which permits any use, distribution and reproduction in any medium, provided the original author(s) and source are credited.

\section{References}

[1] K. Fujikawa and R. Shrock, The Magnetic Moment of a Massive Neutrino and Neutrino Spin Rotation, Phys. Rev. Lett. 45 (1980) 963 [InSPIRE].

[2] P.B. Pal and L. Wolfenstein, Radiative Decays of Massive Neutrinos, Phys. Rev. D 25 (1982) 766 [INSPIRE].

[3] R.E. Shrock, Electromagnetic Properties and Decays of Dirac and Majorana Neutrinos in a General Class of Gauge Theories, Nucl. Phys. B 206 (1982) 359 [InSPIRE].

[4] M. Dvornikov and A. Studenikin, Electric charge and magnetic moment of massive neutrino, Phys. Rev. D 69 (2004) 073001 [hep-ph/0305206] [INSPIRE].

[5] M.S. Dvornikov and A.I. Studenikin, Electromagnetic form-factors of a massive neutrino, J. Exp. Theor. Phys. 99 (2004) 254 [hep-ph/0411085] [INSPIRE].

[6] C. Giunti and A. Studenikin, Neutrino electromagnetic interactions: a window to new physics, Rev. Mod. Phys. 87 (2015) 531 [arXiv: 1403.6344] [INSPIRE].

[7] A.G. Beda et al., The Results of Search for the Neutrino Magnetic Moment in GEMMA Experiment, Adv. High Energy Phys. 2012 (2012) 350150.

[8] B.C. Canas, O.G. Miranda, A. Parada, M. Tortola and J.W.F. Valle, Updating neutrino magnetic moment constraints, Phys. Lett. B 753 (2016) 191 [arXiv:1510.01684] [InSPIRE]. 
[9] Particle Data Group collaboration, C. Patrignani et al., Review of Particle Physics, Chin. Phys. C 40 (2016) 100001 [InSPIRE].

[10] C. Giunti, K.A. Kouzakov, Y.-F. Li, A.V. Lokhov, A.I. Studenikin and S. Zhou, Electromagnetic neutrinos in laboratory experiments and astrophysics, Annalen Phys. 528 (2016) 198 [arXiv : 1506.05387] [INSPIRE].

[11] T.S. Kosmas, O.G. Miranda, D.K. Papoulias, M. Tortola and J.W.F. Valle, Probing neutrino magnetic moments at the Spallation Neutron Source facility, Phys. Rev. D 92 (2015) 013011 [arXiv: 1505.03202] [INSPIRE].

[12] T.S. Kosmas, O.G. Miranda, D.K. Papoulias, M. Tortola and J.W.F. Valle, Sensitivities to neutrino electromagnetic properties at the TEXONO experiment, Phys. Lett. B 750 (2015) 459 [arXiv: 1506 . 08377] [INSPIRE].

[13] C. Buck et al., CONUS: The COhernt NeUtrino Scattering experiment, in preparation.

[14] N.F. Bell, V. Cirigliano, M.J. Ramsey-Musolf, P. Vogel and M.B. Wise, How magnetic is the Dirac neutrino?, Phys. Rev. Lett. 95 (2005) 151802 [hep-ph/0504134] [INSPIRE].

[15] N.F. Bell, M. Gorchtein, M.J. Ramsey-Musolf, P. Vogel and P. Wang, Model independent bounds on magnetic moments of Majorana neutrinos, Phys. Lett. B 642 (2006) 377 [hep-ph/0606248] [INSPIRE].

[16] S. Davidson, M. Gorbahn and A. Santamaria, From transition magnetic moments to Majorana neutrino masses, Phys. Lett. B 626 (2005) 151 [hep-ph/0506085] [INSPIRE].

[17] H.H. Patel, Package-X: A Mathematica package for the analytic calculation of one-loop integrals, Comput. Phys. Commun. 197 (2015) 276 [arXiv: 1503.01469] [INSPIRE].

[18] H. Vogel and J. Redondo, Dark Radiation constraints on minicharged particles in models with a hidden photon, JCAP 02 (2014) 029 [arXiv:1311.2600] [INSPIRE].

[19] R. Essig et al., Working Group Report: New Light Weakly Coupled Particles, arXiv: 1311.0029 [INSPIRE].

[20] R. Foot and S. Vagnozzi, Dissipative hidden sector dark matter, Phys. Rev. D 91 (2015) 023512 [arXiv: 1409.7174] [INSPIRE].

[21] I. Esteban, M.C. Gonzalez-Garcia, M. Maltoni, I. Martinez-Soler and T. Schwetz, Updated fit to three neutrino mixing: exploring the accelerator-reactor complementarity, JHEP 01 (2017) 087 [arXiv: 1611.01514] [INSPIRE].

[22] M. Nemevšek, G. Senjanović and V. Tello, Connecting Dirac and Majorana Neutrino Mass Matrices in the Minimal Left-Right Symmetric Model, Phys. Rev. Lett. 110 (2013) 151802 [arXiv:1211.2837] [INSPIRE].

[23] M. Gozdz, W.A. Kaminski, F. Simkovic and A. Faessler, Transition magnetic moments of Majorana neutrinos in supersymmetry without R-parity in light of neutrino oscillations, Phys. Rev. D 74 (2006) 055007 [hep-ph/0606077] [INSPIRE].

[24] A. Aboubrahim, T. Ibrahim, A. Itani and P. Nath, Large Neutrino Magnetic Dipole Moments in MSSM Extensions, Phys. Rev. D 89 (2014) 055009 [arXiv: 1312.2505] [INSPIRE].

[25] S.M. Barr, E.M. Freire and A. Zee, A Mechanism for large neutrino magnetic moments, Phys. Rev. Lett. 65 (1990) 2626 [INSPIRE].

[26] S.M. Barr and E.M. Freire, Large neutrino magnetic moments and the existence of singly charged scalar fields, Phys. Rev. D 43 (1991) 2989 [INSPIRE]. 
[27] K.S. Babu, D. Chang, W.-Y. Keung and I. Phillips, Comment on 'Mechanism for large neutrino magnetic moments', Phys. Rev. D 46 (1992) 2268 [INSPIRE].

[28] M.B. Voloshin, On Compatibility of Small Mass with Large Magnetic Moment of Neutrino, Sov. J. Nucl. Phys. 48 (1988) 512 [inSPIRE].

[29] R. Barbieri and R.N. Mohapatra, A Neutrino With a Large Magnetic Moment and a Naturally Small Mass, Phys. Lett. B 218 (1989) 225 [InSPIRE].

[30] K.S. Babu and R.N. Mohapatra, Model for Large Transition Magnetic Moment of the $\nu_{e}$, Phys. Rev. Lett. 63 (1989) 228 [INSPIRE].

[31] M. Leurer and N. Marcus, A Model for a Large Neutrino Magnetic Transition Moment and Naturally Small Mass, Phys. Lett. B 237 (1990) 81 [INSPIRE].

[32] D. Chang, W.-Y. Keung, S. Lipovaca and G. Senjanović, Neutrino magnetic moment and the dicyclic group, Phys. Rev. Lett. 67 (1991) 953 [INSPIRE].

[33] G. Ecker, W. Grimus and H. Neufeld, A Light Zeldovich-konopinski-mahmoud Neutrino With a Large Magnetic Moment, Phys. Lett. B 232 (1989) 217 [InSPIRE].

[34] K.S. Babu and R.N. Mohapatra, Supersymmetry and Large Transition Magnetic Moment of the Neutrino, Phys. Rev. Lett. 64 (1990) 1705 [INSPIRE].

[35] D. Chang, W.-Y. Keung and G. Senjanović, Neutrino transitional magnetic moment and nonAbelian discrete symmetry, Phys. Rev. D 42 (1990) 1599 [InSPIRE].

[36] H. Georgi and L. Randall, Charge Conjugation and Neutrino Magnetic Moments, Phys. Lett. B 244 (1990) 196 [INSPIRE].

[37] CMS collaboration, Searches for electroweak production of charginos, neutralinos and sleptons decaying to leptons and $W, Z$ and Higgs bosons in pp collisions at 8 TeV, Eur. Phys. J. C 74 (2014) 3036 [arXiv: 1405.7570] [INSPIRE].

[38] ATLAS collaboration, Search for direct production of charginos, neutralinos and sleptons in final states with two leptons and missing transverse momentum in pp collisions at $\sqrt{s}=8 \mathrm{TeV}$ with the ATLAS detector, JHEP 05 (2014) 071 [arXiv:1403.5294] [INSPIRE].

[39] C. Salazar, R.H. Benavides, W.A. Ponce and E. Rojas, LHC Constraints on 3-3-1 Models, JHEP 07 (2015) 096 [arXiv: 1503.03519] [INSPIRE].

[40] ATLAS collaboration, Evidence for the Higgs-boson Yukawa coupling to tau leptons with the ATLAS detector, JHEP 04 (2015) 117 [arXiv:1501.04943] [INSPIRE].

[41] CMS collaboration, Evidence for the $125 \mathrm{GeV}$ Higgs boson decaying to a pair of $\tau$ leptons, JHEP 05 (2014) 104 [arXiv: 1401.5041] [INSPIRE].

[42] J.-M. Frère, J. Heeck and S. Mollet, Triangle Inequalities for Majorana-Neutrino Magnetic Moments, Phys. Rev. D 92 (2015) 053002 [arXiv:1506.02964] [INSPIRE].

[43] SHIP collaboration, M. Anelli et al., A facility to Search for Hidden Particles (SHiP) at the CERN SPS, arXiv:1504.04956 [INSPIRE]. 